\title{
Pediatric optic neuritis
}

E. Ann Yeh, MD

Jennifer S. Graves, MD

Leslie A. Benson, MD

Evangeline Wassmer, MD

Amy Waldman, MD

Correspondence to

Dr. Yeh:

ann.yeh@sickkids.ca
Supplemental data at Neurology.org

\section{ABSTRACT}

Optic neuritis (ON) is a common presenting symptom in pediatric CNS demyelinating disorders and may be associated with dramatic visual loss. Knowledge regarding clinical presentation, associated diseases, therapy, and outcomes in ON in children has grown over the past decade. These studies have shown that younger children ( $<10$ years of age) are more likely to present with bilateral ON and older children with unilateral ON. Furthermore, studies focusing on visual recovery have shown excellent recovery of high-contrast visual acuity in the majority of children, but functional and structural studies have shown evidence of irreversible injury and functional decline after $\mathrm{ON}$ in children. Although randomized controlled treatment trials have not been performed in children and adolescents with ON, standard of care suggests that the use of highdose pulse steroids is safe and likely effective. This article reviews current knowledge about the clinical presentation and management of pediatric $\mathrm{ON}$, with attention to associated syndromes and evaluative tools that may inform diagnosis and interventions. Neurology ${ }^{\circledR} \mathbf{2 0 1 6 ; 8 7}$ (Suppl 2): S53-S58

\section{GLOSSARY}

ADEM = acute disseminating encephalomyelitis; $\mathbf{A Q P 4}=$ aquaporin-4; $\mathrm{HCVA}=$ high-contrast $\mathrm{VA} ; \mathbf{C R I O N}=$ chronic relapsing inflammatory optic neuropathy; IVIg = IV immunoglobulin; LCLA = low-contrast letter acuity; LCVA = low-contrast VA; MOG = myelin oligodendrocyte glycoprotein; $\mathbf{M S}=$ multiple sclerosis; $\mathbf{N M O}=$ neuromyelitis optica; NMOSD = NMO spectrum disorder; $\mathbf{O C T}=$ optical coherence tomography; $\mathbf{O N}=$ optic neuritis; ONTT = Optic Neuritis Treatment Trial; RNFL = retinal nerve fiber layer; TPE $=$ therapeutic plasma exchange; $\mathbf{V A}=$ visual acuity; $\mathbf{V E P}=$ visual evoked potential; $\mathbf{V F}=$ visual field.

Optic neuritis $(\mathrm{ON})$ occurs in approximately one-fourth of children presenting with an initial demyelinating event. ${ }^{1}$ Although idiopathic $\mathrm{ON}$ may occur in isolation in children, it also exists in conjunction with multifocal inflammatory conditions of the CNS such as acute disseminated encephalomyelitis (ADEM), multiple sclerosis (MS), or neuromyelitis optica (NMO). In the past decade, a number of studies evaluating clinical outcomes of pediatric $\mathrm{ON}$ have been published. These studies have addressed key questions regarding frequency, risk for recurrence, and severity of functional and structural deficits following $\mathrm{ON}$ in children and adolescents. This article reviews current knowledge about definitions and epidemiology of $\mathrm{ON}$ in childhood followed by a discussion of an approach to the evaluation of suspected ON in childhood, treatment, and outcomes.

EPIDEMIOLOGY, DEFINITIONS, AND PRESENTATION ON is one of the most common symptoms of acquired demyelinating syndromes in childhood. The incidence in Canada has been calculated to be 0.2 per 100,000 (95\% confidence interval 0.16-0.3), ${ }^{1}$ but such estimates are not available in other countries for comparison. $\mathrm{ON}$ can be defined as a pathologic process whereby inflammation of one or both optic nerves leads to visual dysfunction. The cardinal features of $\mathrm{ON}$ include decreased visual acuity (VA), dyschromatopsia (abnormal color vision, notably red color desaturation), and visual field (VF) deficits. Visual loss usually occurs over hours to days, typically reaching a nadir within several days of onset. Unilateral presentation at onset may be followed rapidly by bilateral involvement (within 2 weeks: "bilateral simultaneous ON," within 2-12 weeks: "bilateral sequential ON," after 12 weeks: "bilateral recurrent ON"). ${ }^{2}$ In a meta-analysis of published cases of

From the Division of Neurology (E.A.Y.), Hospital for Sick Children, Hospital for Sick Children Research Institute, Department of Pediatrics, University of Toronto, Ontario, Canada; Department of Neurology (J.S.G.), University of California, San Francisco; Department of Neurology (L.A.B.), Boston Children's Hospital, Boston, MA; Department of Neurology (E.W.), Birmingham Children's Hospital, Birmingham, UK; and Department of Neurology (A.W.), The Children's Hospital of Philadelphia, PA.

Go to Neurology.org for full disclosures. Funding information and disclosures deemed relevant by the authors, if any, are provided at the end of the article. 
pediatric $\mathrm{ON}, 72 \%$ of children $<10$ years of age were found to present with bilateral $\mathrm{ON}$, whereas $70 \%$ of children $\geq 10$ years presented with a unilateral clinical event $(p<0.001){ }^{3}$

Pain with eye movement is reported in 33\%-77\% of pediatric cases ${ }^{4-7}$ and therefore does not consistently differentiate inflammatory from noninflammatory optic neuropathies in children. Of importance, children may have difficulty differentiating pain behind the eyes from headache: headache was reported in $53 \%$ of children in one study. ${ }^{7}$

About $20 \%$ of children with ON have a VA of 20/ 40 or better at presentation, another $20 \%$ have a VA between 20/50 and 20/190, and about 60\% have a VA of 20/200 or worse. ${ }^{2,7}$ In some children, central high-contrast VA may remain relatively preserved, but low-contrast vision, color perception, and VF deficits may be prominent. Physical examination findings also include the presence of a relative afferent pupillary defect, with funduscopic examination often revealing optic nerve abnormalities including papillitis in the acute stage and optic nerve pallor in chronic stages. Papillitis has been noted in 46\%$69 \%{ }^{6,8}$ of children with ON compared to one-third of those in the adult Optic Neuritis Treatment Trial (ONTT). ${ }^{9}$

Although MRI is not required to diagnose $\mathrm{ON}$ in children, it may show focal abnormalities of the anterior visual pathway. Typical MRI findings in dedicated orbital MRI studies consist of thickening of the optic nerves on T1-weighted imaging, bright T2 signal along the optic nerve or chiasm, and postgadolinium enhancement. Longitudinally extensive lesions of the optic nerve have been shown to be present in children who are positive for the aquaporin-4 (AQP4) IgG (NMO IgG) and myelin oligodendrocyte glycoprotein (MOG) antibodies (see "Neuromyelitis optica spectrum disorders in children and adolescents," p. S59, for more extensive discussion on MOG antibodies) and not in children with MS. ${ }^{10}$ However, others have found that the longitudinal extent of the lesion does not differ between children with MS and those with ON with non-MS diagnoses. ${ }^{11}$

Computerized VF testing (perimetry) is often pursued in children older than 7 years ${ }^{12}$ using pediatric-specific protocols, whereas adult protocols are generally reliable in those older than 13 years. ${ }^{13}$ The rate of VF defects in acute $\mathrm{ON}$ in children is unknown, but they occur in $97.5 \%$ of adult ON. ${ }^{14}$

In children, prolonged P100 latencies on visual evoked potential (VEP) testing are frequent in the acute phase, ${ }^{15}$ but it is unclear whether children, who may have excellent recovery from $\mathrm{ON},{ }^{16}$ are more likely than adults to have normalized VEP waveforms in long-term follow-up. ${ }^{17}$ Of importance, although $\mathrm{ON}$ may be clinically limited to one eye in many cases, electrophysiologic studies of pediatric MS suggest that bilateral involvement occurs frequently in pediatric MS even in the absence of overt $\mathrm{ON}$ in the fellow eye. ${ }^{18}$

FUNCTIONAL AND STRUCTURAL OUTCOMES The majority $(58 \%-97 \%)$ of children have been found to experience full high-contrast visual acuity (HCVA) recovery (better than $20 / 30$ or 20/40) in separate observational US ( $\mathrm{n}=15$ and $\mathrm{n}=29)$, Canadian $(\mathrm{n}=36)$, and UK $(\mathrm{n}=44)$ cohorts. $^{2,7,16,19}$ Although recovery of HCVA is excellent, children often report subjective visual abnormalities after ON that HCVA cannot capture, likely secondary to injury to the optic nerve, including axonal degeneration.

Optical coherence tomography (OCT) can provide assessments of neuronal injury, including retinal nerve fiber layer (RNFL) thickness and retinal ganglion cell body and axon layers. In pediatric MS, eyes with a history of $\mathrm{ON}$ have been reported to have $10 \%-20 \%$ thinner RNFL than MS non-ON eyes or control eyes and 6\% lower macular volumes. ${ }^{20-22}$ Using time-domain OCT, severe RNFL thinning may occur after a single episode of $\mathrm{ON}$ in certain cases, including $\mathrm{ON}$ associated with transverse myelitis and ADEM (67 microns) vs MS (83 microns) and healthy age- and sex-matched controls $(107 \pm 12$ microns), suggesting potentially different pathophysiologic processes driving these entities. ${ }^{22}$ In recurrent disease in children, stepwise decrease in RNFL (8-10 microns) may be seen following each episode of ON. ${ }^{21}$

Other sensitive tools for the measurement of visual dysfunction are metrics of low-contrast dysfunction, contrast sensitivity, and low-contrast letter acuity (LCLA). ${ }^{14,23,24}$ Three pediatric studies have demonstrated decreased low-contrast visual acuity (LCVA) after ON. ${ }^{21,22,25}$ One study showed a correlation between RNFL thickness and LCVA (using 2.5\% Sloan letter charts) for all demyelinating disease eyes $(R=0.42, p=0.002) .{ }^{22}$ In contrast, another study demonstrated that LCVA was not associated with RNFL thinning after adjusting for age and intereye correlations. ${ }^{25}$ Decreases in other functional testing, including color vision and VF, correlate with decreases in RNFL. Replication of these results in other cohorts is needed. ${ }^{21}$

DIFFERENTIAL DIAGNOSIS As previously noted, $\mathrm{ON}$ in childhood is marked by heterogeneity and may occur as a monophasic illness, recurrent isolated $\mathrm{ON}$, and recurrent $\mathrm{ON}$ in the context of multifocal inflammatory CNS disease. In the child presenting with a first-time episode of acute or subacute visual loss attributed to the optic nerve, once infectious, genetic, and neoplastic etiologies have been ruled out, specific clinical and laboratory indicators may assist in predicting likelihood of $\mathrm{ON}$ being the 
first manifestation of MS, chronic relapsing inflammatory optic neuropathy (CRION), NMO spectrum disorder (NMOSD), or part of an underlying systemic rheumatologic condition such as systemic lupus erythematosus. Accurate diagnosis, with a focus on potential etiologies, is key to decision-making around acute and chronic therapy. ${ }^{26,27}$ History, physical examination, and laboratory investigations should be oriented toward extent of visual loss as well as evaluation for involvement of other areas of the CNS and systemic involvement, including ocular, dermatologic, joint, renal, and hepatic involvement. Of importance, the presence of other ocular findings, including significant retinal involvement and inflammatory cells in the vitreous, should alert the clinician to the need for a broader rheumatologic workup. Differential considerations include sarcoidosis, CNS lupus, small vessel CNS vasculitis, and idiopathic granulomatous optic neuropathy (figure). Unusual features of the history or physical examination, including insidious onset, painless visual loss, or severe optic nerve pallor at presentation, should lead to consideration of diagnostic possibilities such as compressive lesions; posterior scleritis; maculopathies; retinopathies; ischemic optic neuropathy; toxic, nutritional, and infectious etiologies; and genetic optic atrophies (e.g., mitochondrial disorders, such as Leber hereditary optic neuropathy). Although organic etiologies should be ruled out first in children with visual complaints, inconsistencies in history and examination may suggest a nonorganic/ functional etiology. ${ }^{28}$ In these cases, involvement of counselors, psychiatrists, and other therapists may be necessary to return the child to baseline functioning.

RISK OF RECURRENT CNS DISEASE AFTER ACUTE ON Between $13 \%$ and $36 \%$ of children who present with an initial episode of $\mathrm{ON}$ are eventually diagnosed with MS after a short follow-up period. ${ }^{2,5,8}$ Likelihood of $\mathrm{ON}$ being the first

Figure Algorithim for the approach to a child presenting with acute optic neuritis

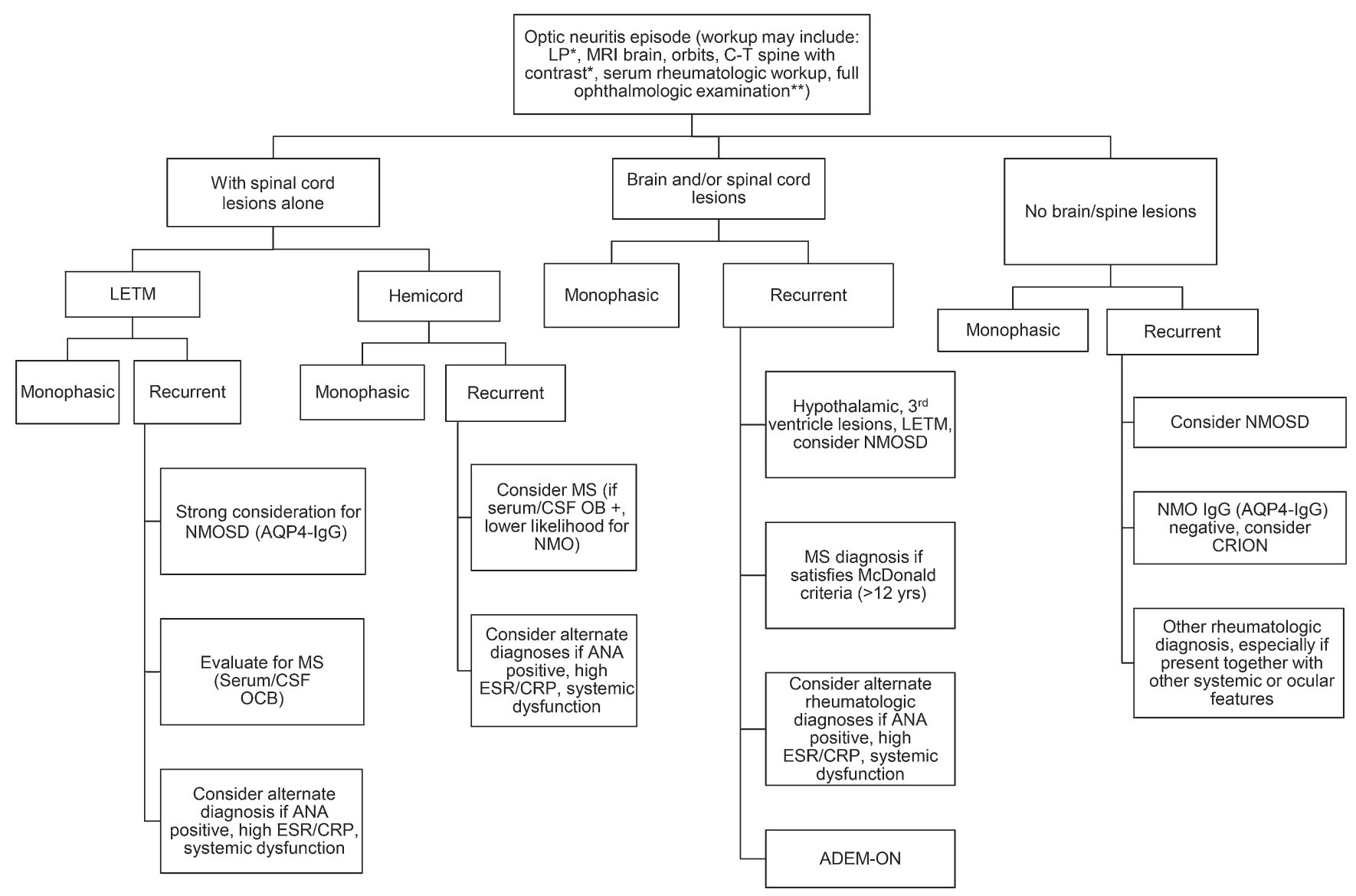

*Lumbar puncture (LP) and MRI/CT spine to be performed at clinician's discretion. These investigations may be particularly useful in the presence of brain lesions. **Full ophthalmologic examination should be performed to exclude other ocular inflammatory conditions (e.g., uveitis, scleritis, episcleritis, or retinal vasculopathy), which may broaden the differential diagnosis to include systemic rheumatologic processes. ADEM = acute disseminated encephalomyelitis; $\mathrm{ANA}=$ antinuclear antibody; $\mathrm{AQP} 4=$ aquaporin-4; CRION = chronic relapsing inflammatory optic neuropathy; $\mathrm{CRP}=\mathrm{C}$-reactive protein; $\mathrm{ESR}=\mathrm{erythrocyte}$ sedimentation rate; LETM = longitudinally extensive transverse myelitis; MS = multiple sclerosis; NMO = neuromyelitis optica; NMOSD = NMO spectrum disorder; $\mathrm{OCB}=$ oligoclonal band; $\mathrm{ON}=$ optic neuritis. 
manifestation of MS is high in children with white matter lesions on brain MRI.,8,19 Conversely, confirmation of an MS diagnosis after a single episode of $\mathrm{ON}$ in childhood with no brain lesions is infrequent. In one prospective study (3 years follow-up), only $1.9 \%$ of children experiencing acute demyelination with no brain lesions were later confirmed to have MS. ${ }^{29}$ CSF markers may help to establish risk for MS in the setting of an isolated $\mathrm{ON}$ event. CSF oligoclonal bands were found in $80 \%$ of pediatric patients with MS and in only $15 \%$ of children with monophasic ON. ${ }^{30}$

Twenty-six percent of patients with adult MS and $42 \%-58 \%$ of patients with NMO may initially present with $\mathrm{ON}$ alone or in combination with other neurologic deficits. ${ }^{31,32}$ Although young children may have bilateral severe $\mathrm{ON}$ without experiencing recurrence, given the importance of treatment to prevent irreversible visual disability in NMOSD and the high association of serologic markers such as ANA and anti-Ro/SSA with NMOSD, severe bilateral $\mathrm{ON}$, poor response to steroids, or the presence of brain or spinal cord MRI findings typical of NMOSD should lead to evaluation of serum for AQP4 antibodies and concomitant autoantibodies. ${ }^{26}$ The clinical significance of other antibodies and rheumatologic markers is unclear: whether the $\mathrm{ON}$ is a manifestation of an underlying rheumatologic condition with concomitant AQP-4 IgG positivity is unknown. However, previous studies have suggested high specificity of AQP-4 IgG for the diagnosis of NMO, so AQP4IgG positivity with suggestive clinical manifestations is supportive of the diagnosis of NMOSD. ${ }^{33}$ This is addressed in the review of NMOSD contained in this supplement.

More recently, the entity of ADEM followed by ON (ADEM-ON) has been identified. ${ }^{34}$ Currently, active interest in the connection between serum antiMOG antibodies and recurrent ON and ADEM-ON in children exists, but there is uncertainty about this relationship. ${ }^{35}$ The pathologic significance of antiMOG antibodies has yet to be defined. This remains an evolving field.

Recurrence of $\mathrm{ON}$ in the absence of accompanying associated brain or spinal cord involvement may also occur in children. This is an uncommon phenomenon, representing 6/94 patients $(5 \%)$ in a large series of consecutively followed children in a Canadian center. ${ }^{36}$ Some of these patients may present with steroid-dependent relapses, similar to adults with CRION. ${ }^{37}$ Although in the past CRION has been considered to be on the NMO spectrum, this entity can be distinguished from NMO by following revised diagnostic criteria for CRION: at least 2 episodes of $\mathrm{ON}$, objective loss of visual function, negative testing for NMO IgG, contrast enhancement of the affected optic nerve, and response to immunosuppressive treatment with relapse on withdrawal. ${ }^{37}$ If CRION is suspected, gradual steroid taper and prompt treatment including consideration of immunosuppressive agents are recommended, as visual outcome may be poor. ${ }^{37}$

TREATMENT No clinical trials have been performed for pediatric $\mathrm{ON}$, so clinical practice currently follows evidence gleaned from the ONTT, ${ }^{38,39}$ in which recovery after IV steroid administration was more rapid than after placebo or oral steroids in the first 15 days after onset. By 7 weeks after onset, little difference between the groups could be seen. ${ }^{38}$ Systematic reviews have suggested no long-term difference in HCVA in adults treated acutely with steroids compared to those who were not treated, although analysis of other visual outcomes was not included in these studies. ${ }^{40}$

Treatment in the pediatric population consists of $30 \mathrm{mg} / \mathrm{kg}$ per day IV methylprednisolone, maximum $1 \mathrm{~g}$ daily, for 3-5 days. The need for a prolonged course of oral steroids is unknown: one retrospective study suggested no difference in outcome between a shorter ( 2 weeks) and longer ( $>2$ weeks) course of steroids in children with acute ON. ${ }^{41} \mathrm{ON}$ treatment with pulse steroids may be particularly relevant for children, as speeding recovery from 7 to 2 weeks may prevent key psychosocial challenges, including the need to make up schoolwork, and other sequelae resulting from functional visual limitations.

Little evidence for acute therapies beyond steroid treatment exists. However, if improvement does not occur after administration of steroids, a second course of high-dose steroids, therapeutic plasma exchange (TPE), immunoadsorption, or IV immunoglobulin (IVIg) may be considered. Cases series have reported benefit with TPE and immunoadsorption in acute $\mathrm{ON}$ in adults. ${ }^{42,43}$ Likewise, case series suggest that plasmapheresis is safe in pediatric demyelination ${ }^{44}$ and has potential benefit in steroid-resistant pediatric ON. ${ }^{45}$

Although some observational studies suggested a possible benefit to the use of IVIg in corticosteroid-refractory acute $\mathrm{ON}$ in adults, ${ }^{46}$ a double-blind trial showed no difference between outcomes when comparing IVIg and placebo in acute ON. ${ }^{47}$ Evidence for the use of IVIg in pediatric $\mathrm{ON}$ is limited to case reports and small case series.

PROPHYLACTIC THERAPY IN RECURRENT DISEASE Growing knowledge suggesting that $\mathrm{ON}$ can be a first manifestation of disorders such as MS and other systemic autoimmune conditions exists. In cases in which underlying NMO or rheumatologic disorders are suspected, treatment with steroids and chronic immunotherapy may prevent or delay the 
appearance of further inflammatory CNS events. Choice of prophylactic therapy for recurrent disease (MS/NMO/systemic rheumatologic disease) is dictated by the underlying diagnosis. Reports of the use of immunosuppressive therapy in CRION (e.g., mycophenolate mofetil, azathioprine, rituximab, cyclophosphamide, and steroids, among other therapies) have been published, although efficacy has not been established. ${ }^{37}$ Efficacy of prophylactic treatment for ADEM-ON is unknown. ${ }^{34}$

CONCLUSION ON is a common presenting symptom in pediatric CNS demyelinating disorders. Clinical symptoms may be dramatic, especially in younger children, with severe decreases in visual acuity in more than half of children presenting with $\mathrm{ON}$ and a high likelihood of bilateral disease. Although recurrent disease may occur in approximately one-third of patients, risk stratification for recurrence is informed by the presence or absence of concomitant brain MRI T2-hyperintense lesions. Close attention should be paid to potential underlying etiologies, including rheumatologic and other antibody-mediated etiologies. Therapeutic intervention with steroids, while unlikely to change the medical outcomes in the majority of cases, may be of benefit in children, given the rapid response to steroids and high likelihood of returning to baseline within 1-2 weeks instead almost 2 months (without such treatment). Plasmapheresis and IVIg may be used in the event of poor response to steroids, especially in children with NMOSD.

Although outcome studies suggest a return to almost-normal HCVA in the majority of children, other studies have demonstrated early and marked injury to the optic nerve in pediatric $\mathrm{ON}$ after the first event together with decreases in functional testing, including LCLA and VF testing. Future research should focus on the relationship between functional and patient-related outcomes, optic nerve injury, and structural changes in other areas of the CNS in children with demyelinating disorders, as well as on the evaluation of progressive change in optic nerve head, retinal ganglion cell, and RNFL thickness in this population.

\section{AUTHOR CONTRIBUTIONS}

All authors contributed to manuscript drafting and critical revision of the manuscript for important intellectual content. All authors approved the manuscript for submission.

\section{STUDY FUNDING}

This supplement is made possible by funding from the MS Cure Fund, Danish MS Society, German MS Society, Italian MS Association, MS International Federation, MS Research Foundation (Netherlands), National MS Society (USA) and Swiss MS Society.

\section{DISCLOSURE}

E. A. Yeh receives funding from the National MS Society, the Canadian Institutes of Health Research, the Mario Batali Foundation, the Dairy
Farmers of Ontario, SickKids Foundation, SickKids Innovation Fund, CMSMS/PHAC, the Canadian Multiple Sclerosis Scientific Research Foundation, and the MS Society of Canada. J. Graves reports no disclosures relevant to the manuscript. L. Benson receives funding from Boston Children's Hospital Faculty Career Development fellowship and National MS Society. E. Wassmer received honoraria for speaking/travel costs from BiogenIdec, Teva, Genzyme, Shire, UCB, and Merck Serono. She is an investigator in trials with BiogenIdec, Sanofi, and Novartis. Her MS research projects have been funded by the UK MS Society, Action Medical Research, and Birmingham Children's Hospital Research Foundation. A. Waldman receives funding from the NIH (NINDS K23), NMSS, Novartis, and Biogen. Go to Neurology.org for full disclosures.

Received August 19, 2015. Accepted in final form January 12, 2016.

\section{REFERENCES}

1. Banwell B, Kennedy J, Sadovnick D, et al. Incidence of acquired demyelination of the CNS in Canadian children. Neurology 2009;72:232-239.

2. Bonhomme GR, Waldman AT, Balcer LJ, et al. Pediatric optic neuritis: brain MRI abnormalities and risk of multiple sclerosis. Neurology 2009;72:881-885.

3. Waldman AT, Stull LB, Galetta SL, Balcer LJ, Liu GT. Pediatric optic neuritis and risk of multiple sclerosis: meta-analysis of observational studies. J AAPOS 2011; 15:441-446.

4. Alper G, Wang L. Demyelinating optic neuritis in children. J Child Neurol 2009;24:45-48.

5. Lucchinetti CF, Kiers L, O'Duffy A, et al. Risk factors for developing multiple sclerosis after childhood optic neuritis. Neurology 1997;49:1413-1418.

6. Lana-Peixoto MA, Andrade GC. The clinical profile of childhood optic neuritis. Arq Neuropsiquiatr 2001;59: 311-317.

7. Wilejto M, Shroff M, Buncic JR, Kennedy J, Goia C, Banwell B. The clinical features, MRI findings, and outcome of optic neuritis in children. Neurology 2006;67:258-262.

8. Morales DS, Siatkowski RM, Howard CW, Warman R. Optic neuritis in children. J Pediatr Ophthalmol Strabismus 2000;37:254-259.

9. Bonhomme GR, Mitchell EB. Treatment of pediatric optic neuritis. Curr Treatment Options Neurol 2012;14: 93-102.

10. Ramanathan S, Prelog K, Barnes EH, et al. Radiological differentiation of optic neuritis with myelin oligodendrocyte glycoprotein antibodies, aquaporin- 4 antibodies, and multiple sclerosis. Mult Scler 2016;22:470-482.

11. Graves J, Kraus V, Soares BP, Hess CP, Waubant E. Longitudinally extensive optic neuritis in pediatric patients. J Child Neurol 2015;30:120-123.

12. Morales J, Brown SM. The feasibility of short automated static perimetry in children. Ophthalmology 2001;108:157-162.

13. Wabbels BK, Wilscher S. Feasibility and outcome of automated static perimetry in children using continuous light increment perimetry (CLIP) and fast threshold strategy. Acta Ophthalmol Scand 2005;83:664-669.

14. The clinical profile of optic neuritis. Experience of the Optic Neuritis Treatment Trial. Optic Neuritis Study Group. Arch Ophthalmol 1991;109:1673-1678.

15. Voitenkov V, Skripchenko N, Klimkin A. Visual pathways involvement in clinically isolated syndrome in children. Int J Ophthalmol 2015;8:382-384.

16. Malik MT, Healy BC, Benson LA, et al. Factors associated with recovery from acute optic neuritis in patients with multiple sclerosis. Neurology 2014;82:2173-2179. 
17. Heussinger N, Kontopantelis E, Rompel O, Paulides M, Trollmann R. Predicting multiple sclerosis following isolated optic neuritis in children. Eur J Neurol 2013;20: 1292-1296.

18. Pohl D, Rostasy K, Treiber-Held S, Brockmann K, Gartner J, Hanefeld F. Pediatric multiple sclerosis: detection of clinically silent lesions by multimodal evoked potentials. J Pediatr 2006;149:125-127.

19. Absoud M, Cummins C, Desai N, et al. Childhood optic neuritis clinical features and outcome. Arch Disease Childhood 2011;96:860-862.

20. Yilmaz U, Gucuyener K, Erin DM, et al. Reduced retinal nerve fiber layer thickness and macular volume in pediatric multiple sclerosis. J Child Neurol 2012;27:1517-1523.

21. Yeh EA, Marrie RA, Reginald YA, et al. Functional-structural correlations in the afferent visual pathway in pediatric demyelination. Neurology 2014;83:2147-2152.

22. Yeh EA, Weinstock-Guttman B, Lincoff N, et al. Retinal nerve fiber thickness in inflammatory demyelinating diseases of childhood onset. Mult Scler 2009;15:802-810.

23. Baier ML, Cutter GR, Rudick RA, et al. Low-contrast letter acuity testing captures visual dysfunction in patients with multiple sclerosis. Neurology 2005;64:992-995.

24. Balcer LJ, Baier ML, Pelak VS, et al. New low-contrast vision charts: reliability and test characteristics in patients with multiple sclerosis. Mult Scler 2000;6:163-171.

25. Waldman AT, Hiremath G, Avery RA, et al. Monocular and binocular low-contrast visual acuity and optical coherence tomography in pediatric multiple sclerosis. Mult Scler Relat Disord 2013;3:326-334.

26. Pittock SJ, Lennon VA, de Seze J, et al. Neuromyelitis optica and non organ-specific autoimmunity. Arch Neurol 2008;65:78-83

27. Petzold A, Wattjes MP, Costello F, et al. The investigation of acute optic neuritis: a review and proposed protocol. Nat Reviews Neurol 2014;10:447-458.

28. Fernandez Carbonell C, Benson L, Rintell D, Prince J, Chitnis T. Functional relapses in pediatric multiple sclerosis. J Child Neurol 2014;29:943-946.

29. Verhey LH, Branson HM, Shroff MM, et al. MRI parameters for prediction of multiple sclerosis diagnosis in children with acute CNS demyelination: a prospective national cohort study. Lancet Neurol 2011;10:1065-1073.

30. Heussinger N, Kontopantelis E, Gburek-Augustat J, et al. Oligoclonal bands predict multiple sclerosis in children with optic neuritis. Ann Neurol 2015;77:1076-1082.

31. Chitnis T, Glanz B, Jaffin S, Healy B. Demographics of pediatric-onset multiple sclerosis in an MS center population from the Northeastern United States. Mult Scler 2009;15:627-631.

32. Collongues $\mathrm{N}$, Marignier $\mathrm{R}$, Zephir $\mathrm{H}$, et al. Long-term follow-up of neuromyelitis optica with a pediatric onset. Neurology 2010;75:1084-1088.
33. Wingerchuk DM, Banwell B, Bennett JL, et al. International consensus diagnostic criteria for neuromyelitis optica spectrum disorders. Neurology 2015;85:177-189.

34. Huppke P, Rostasy K, Karenfort M, et al. Acute disseminated encephalomyelitis followed by recurrent or monophasic optic neuritis in pediatric patients. Mult Scler 2013; 19:941-946.

35. Kitley J, Leite MI, Nakashima I, et al. Prognostic factors and disease course in aquaporin- 4 antibody-positive patients with neuromyelitis optica spectrum disorder from the United Kingdom and Japan. Brain 2012;135:18341849.

36. Lai CY, Yeh E. Recurrent isolated optic neuritis in children. Ann Neurol 2013;74:S152.

37. Petzold A, Plant GT. Chronic relapsing inflammatory optic neuropathy: a systematic review of 122 cases reported. J Neurol 2014;261:17-26.

38. Beck RW, Cleary PA, Anderson MM Jr, et al. A randomized, controlled trial of corticosteroids in the treatment of acute optic neuritis. The Optic Neuritis Study Group. N Engl J Med 1992;326:581-588.

39. Beck RW, Cleary PA, Trobe JD, et al. The effect of corticosteroids for acute optic neuritis on the subsequent development of multiple sclerosis. The Optic Neuritis Study Group. N Engl J Med 1993;329:1764-1769.

40. Gal RL, Vedula SS, Beck R. Corticosteroids for treating optic neuritis. Cochrane Database Syst Rev 2015;8: CD001430.

41. Jayakody H, Bonthius DJ, Longmuir R, Joshi C. Pediatric optic neuritis: does a prolonged course of steroids reduce relapses? A preliminary study. Pediatr Neurol 2014;51: 721-725.

42. Ruprecht K, Klinker E, Dintelmann T, Rieckmann P, Gold R. Plasma exchange for severe optic neuritis: treatment of 10 patients. Neurology 2004;63:1081-1083.

43. Koziolek MJ, Tampe D, Bahr M, et al. Immunoadsorption therapy in patients with multiple sclerosis with steroid-refractory optical neuritis. J Neuroinflammation 2012;9:80.

44. Bigi S, Banwell B, Yeh EA. Outcomes after early administration of plasma exchange in pediatric central nervous system inflammatory demyelination. J Child Neurol 2015; 30:874-880.

45. Koziolek M, Muhlhausen J, Friede T, et al. Therapeutic apheresis in pediatric patients with acute CNS inflammatory demyelinating disease. Blood Purif 2013;36:92-97.

46. Tselis A, Perumal J, Caon C, et al. Treatment of corticosteroid refractory optic neuritis in multiple sclerosis patients with intravenous immunoglobulin. Eur J Neurol 2008;15:1163-1167.

47. Roed HG, Langkilde A, Sellebjerg F, et al. A double-blind, randomized trial of IV immunoglobulin treatment in acute optic neuritis. Neurology 2005;64:804-810. 


\title{
Neurology
}

\author{
Pediatric optic neuritis \\ E. Ann Yeh, Jennifer S. Graves, Leslie A. Benson, et al. \\ Neurology 2016;87;S53-S58 \\ DOI 10.1212/WNL.0000000000002822
}

This information is current as of August 29, 2016

\section{Updated Information \& Services}

\section{References}

Citations

Subspecialty Collections

\section{Permissions \& Licensing}

Reprints including high resolution figures, can be found at: http://n.neurology.org/content/87/9_Supplement_2/S53.full

This article cites 47 articles, 0 of which you can access for free at: http://n.neurology.org/content/87/9_Supplement_2/S53.full\#ref-list-1

This article has been cited by 1 HighWire-hosted articles: http://n.neurology.org/content/87/9_Supplement_2/S53.full\#\#otherartic les

This article, along with others on similar topics, appears in the following collection(s):

All Demyelinating disease (CNS)

http://n.neurology.org/cgi/collection/all_demyelinating_disease_cns All Pediatric

http://n.neurology.org/cgi/collection/all_pediatric

Multiple sclerosis

http://n.neurology.org/cgi/collection/multiple_sclerosis

Optic neuritis; see Neuro-ophthalmology/Optic Nerve

http://n.neurology.org/cgi/collection/optic_neuritis

Information about reproducing this article in parts (figures,tables) or in its entirety can be found online at:

http://www.neurology.org/about/about_the_journal\#permissions

Information about ordering reprints can be found online: http://n.neurology.org/subscribers/advertise

Neurology ${ }^{\circledR}$ is the official journal of the American Academy of Neurology. Published continuously since 1951, it is now a weekly with 48 issues per year. Copyright () 2016 American Academy of Neurology. All rights reserved. Print ISSN: 0028-3878. Online ISSN: 1526-632X.

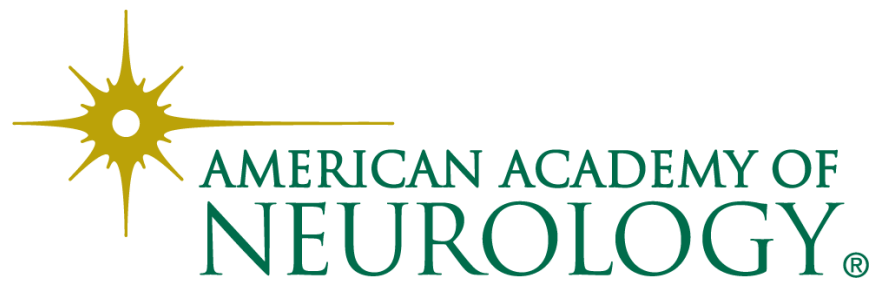

